\title{
Zum 80. Geburtstag von Professor Johann GOLSER
}

\section{Robert Galler}

Lehrstuhl für Subsurface Engineering, Montanuniversität Leoben, Leoben, Österreich

Angenommen 30. Oktober 2020; online publiziert 18. November 2020

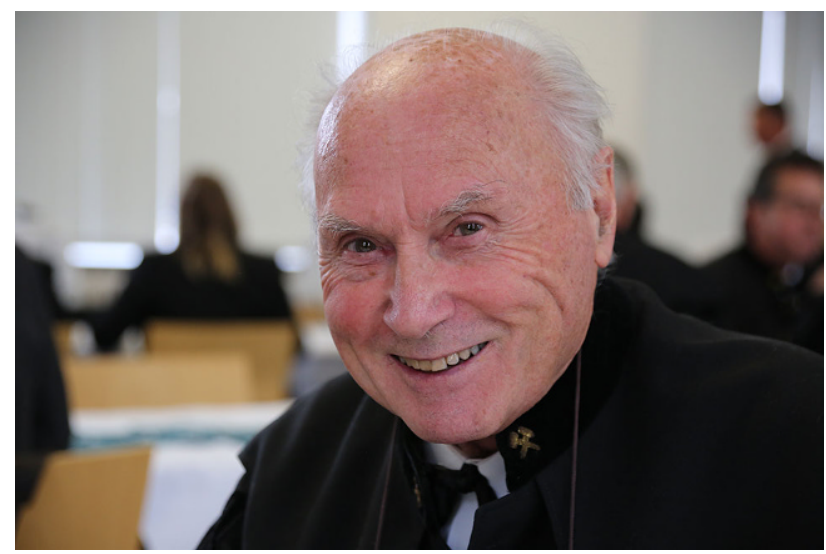

Johann Golser wurde am 27. Mai 1940 in Villach geboren und verbrachte seine Jugendzeit in Spittal an der Drau. Sein beruflicher Weg in das Bauwesen war ihm vielleicht schon in die Wiege gelegt, hat sich doch sein Vater, der nach einer Arsenvergiftung den Beruf des Stuckateurs aufgeben musste, vom Maurer bis zum Bauleiter hinaufgearbeitet.

Der angedachte Bildungsweg für Johann Golser war der damals klassische: Volksschule, Hauptschule, Maurerlehre, Berufsschule, wenn da nicht in der 4. Klasse Volksschule eine entscheidende zufällige Weichenstellung für die weitere Ausbildung erfolgt wäre. Der Klassenlehrer erkrankte ein paar Monate vor Schulschluss. Die Direktorin kam in die Klasse und entschied, dass die Schüler, die ins Gymnasium gehen wollten, zur Vorbereitung für die Aufnahmeprüfung in ihre Klasse übersiedeln sollten. Es folgte eine Schulnachricht an die Eltern. Johann Golser hörte erstmals von dieser Möglichkeit einer höheren Schulbildung und teilte seinen Eltern umgehend mit, dass er ins Gymnasium gehen möchte. (Abb. 1).

\footnotetext{
Univ.-Prof. Dipl.-Ing. Dr. mont. R. Galler ( $\varangle)$

Lehrstuhl für Subsurface Engineering,

Montanuniversität Leoben,

Franz-Josef-Str. 18,

8700 Leoben, Österreich

robert.galler@unileoben.ac.at
}

Es folgten lange, für Johann Golser bis heute belastende Gespräche und Kämpfe, da seine Eltern der Meinung waren, dass das Gymnasium nichts für Arbeiterkinder ist und sich die Familie diese Ausbildung nicht leisten könnte. Letztendlich durfte er das Realgymnasium besuchen und es folgte eine schöne Zeit mit tiefen Freundschaften, sportlichen Aktivitäten mit Bergsteigen, Leichtathletik und Rudern, aber auch viel Arbeit. Naturwissenschaftliche Fächer bereiteten ihm Freude, Latein hingegen war ihm ein Dorn im Auge.

Nach Abschluss des Realgymnasiums studierte Johann Golser an der Technischen Hochschule in Graz Bauingenieurwesen, ein Studium, das er sich großteils über Ferialarbeit und das Geben von Nachhilfestunden selbst finanziert hat.

Im 3. Semester heiratet Johann Golser seine Jugendliebe Sieglinde aus der Gymnasialzeit, mit der er nunmehr seit 60 Jahren verheiratet ist. Herzliche Gratulation an dieser Stelle! Im zweiten Studienabschnitt zieht seine Frau mit ihrem - heute 60 Jahre jungen - Sohn nach Graz.

Es wurde 18 Semester lang gründlich studiert, das feucht-fröhliche Studentenleben in der Studentenstadt Graz genossen und sportlichen Aktivitäten im Akademischen Turnverein nachgegangen, bevor er im Jahr 1967 zum Diplomingenieur graduiert wurde. (Abb. 2).

Im 2. Studienabschnitt musste man zur damaligen Zeit zwei Wahlausbildungen belegen; Johann Golser entschied sich für zwei sehr unterschiedliche, nämlich eine statischkonstruktive Vertiefung und jene in Bodenmechanik und Grundbau. Felsmechanik und Tunnelbau wurde nur in sehr geringem Umfang angeboten; konkret wurde das Fach Felsmechanik in den Lehrveranstaltungen zur Geologie gestreift, Tunnelbau wurde mit einem kleinen Lehrauftrag abgedeckt.

Bei Beendigung des Studiums hatte Johann Golser schon fast beim Amt der Kärntner Landesregierung, Abteilung Brückenbau angeheuert, aber wieder kam durch Zufall eine Weggabelung, an der er sich für einen weiteren Schritt in Richtung Zukunft entscheiden musste. Prof. Christian Veder hat Johann Golser eine Assistenzstelle an seinem Lehrstuhl für Bodenmechanik und Grundbau angeboten. 


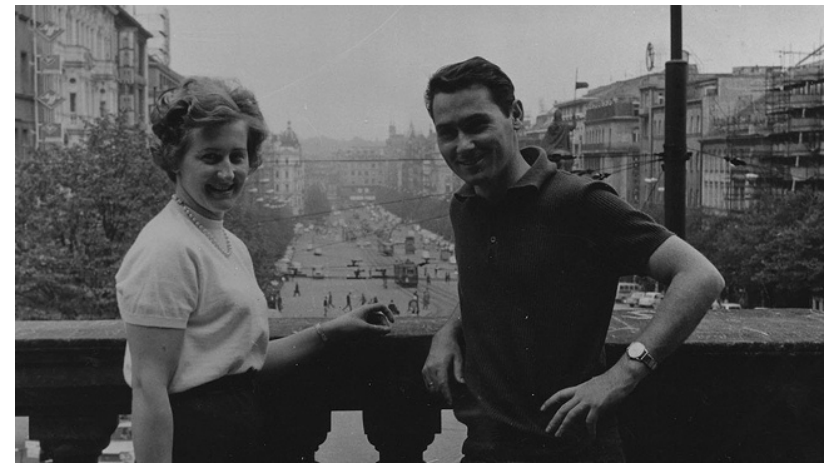

Abb. 1: Johann und Sieglinde Golser

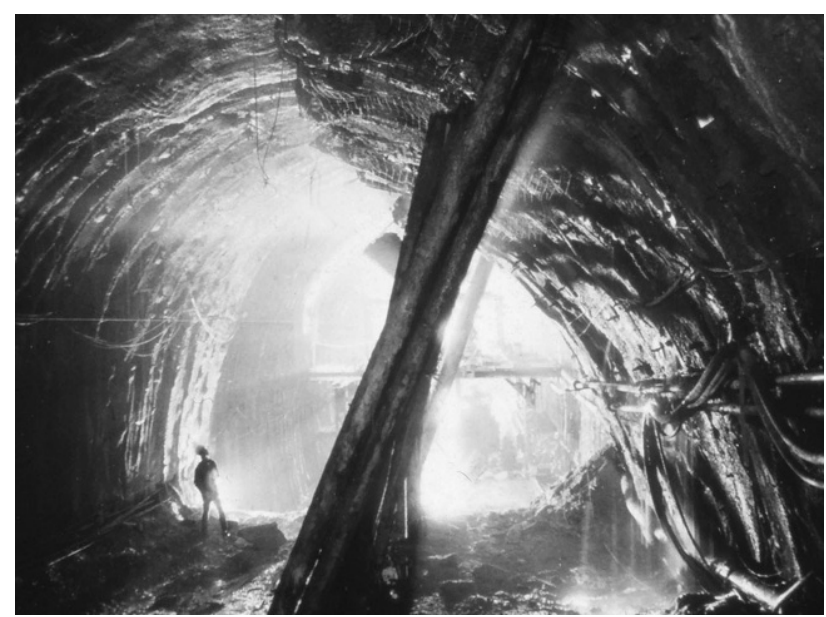

Abb. 3: Tauerntunnel-Scherbruch

Johann Golser hat dieses Angebot jedoch nicht angenommen, da er gleich in die Praxis wollte. Prof. Veder nahm ihm das nicht übel und gab ihm einen entscheidenden Rat. Das Ingenieurbüro Pacher in Salzburg und Prof. Rabcewicz suchten junge Ingenieure für interessante Planungs- und Beratungsarbeiten im Bereich Grundbau, Felsbau und Tunnelbau. Johann Golser wurde umgehend angeheuert und war damit im Kreis der Salzburger Geomechaniker gelandet, aus dem die heutige Österreichische Gesellschaft für Geomechanik hervorging. Seine ersten Aufgaben waren die Mitarbeit bei Projekten der Tauernautobahn, Hangsicherungen, Brückenfundierungen in tiefreichend instabilen Berghängen und Tunnelbauten. (Abb. 3).

Anfangs hatte Johann Golser Zweifel, ob dieser Fachbereich das Richtige für ihn wäre, da es fast aussichtslos schien, in absehbarer Zeit jene Erfahrung zu sammeln, die Prof. Pacher bei der treffsicheren Abschätzung von Kennwerten und beim raschen Finden praktischer Lösungen hatte. Es galt, das Verhalten des Gebirges als Reaktion auf die Eingriffe des Tunnelbaus und Versagensphänomene zu beobachten, und zu versuchen, das Beobachtete zu deuten, rechnerisch zu erfassen und die richtigen Maßnahmen zu treffen.

Die wöchentlichen Heimfahrten nach Kärnten über zwei Alpenpässe - Johann und seine Frau lebten eine Wochen-

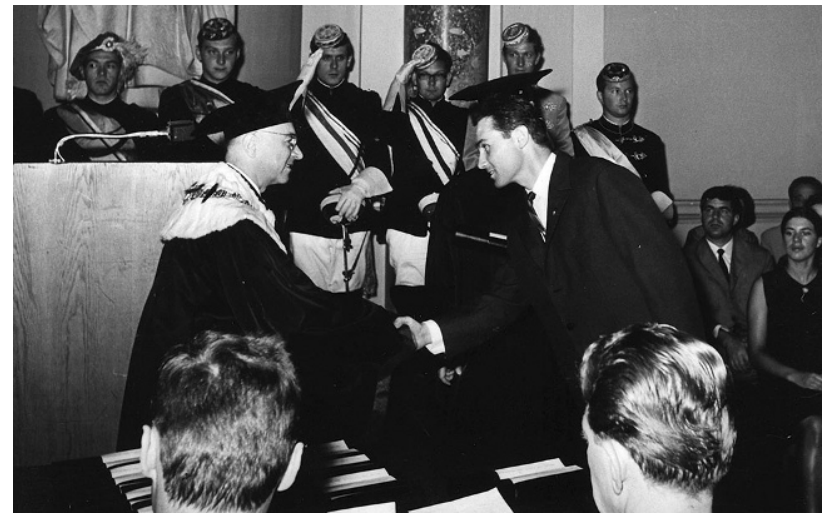

Abb. 2: Graduierung zum Diplomingenieur für Bauwesen an derTU Graz

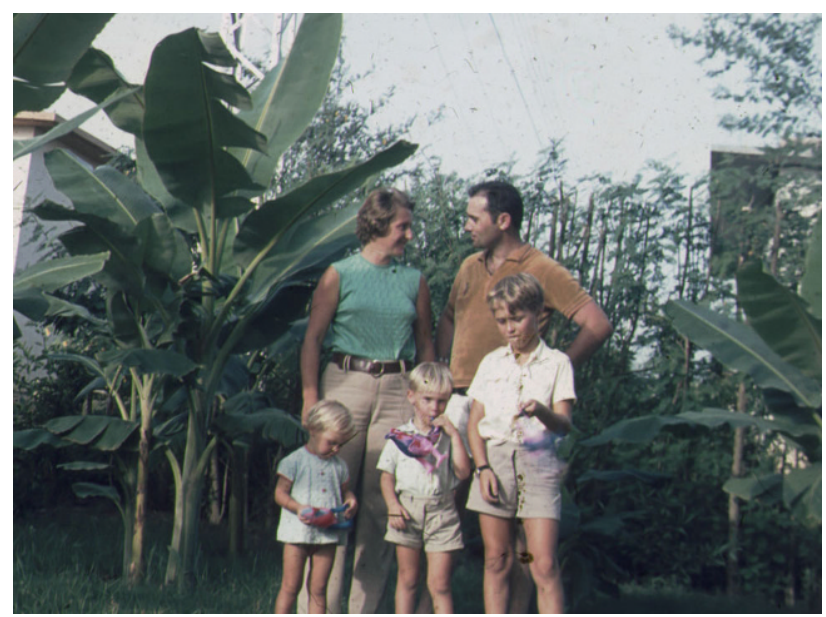

Abb. 4: Familie Golser in Pakistan

end-Ehe - führten entlang der Baustellen der Tauernautobahn. Baustellenbesuche und der Einblick in das praktische Baugeschehen waren für ihn sehr hilfreich.

Prof. Rabcewicz lebte mit seiner Frau in Mauterndorf in einem wunderschönen Landhaus am Fanningberg etwa in der Mitte des Weges zwischen Salzburg und Kärnten, den Johann Golser wöchentlich zurücklegen musste. Projektbesprechungen fanden sehr regelmäßig im schönen Lungau statt. Rabcewicz und seine Frau Elisabeth erzählten immer wieder über ihre oftmals abenteuerlichen Erlebnisse auf Baustellen in der Türkei und in Persien. So entwickelte sich für das spätere Berufsleben Johann Golsers eine bestimmende, enge und freundschaftliche Zusammenarbeit mit Prof. Rabcewicz, dem Begründer der NATM. Ausführliche, oft bis spät in die Nacht dauernde Fachsimpeleien waren mit ein Grund, dass Johann Golser sich schließlich ganz der Geomechanik und dem Tunnelbau verschrieb.

Rabcewicz hat Golser gelehrt, das Gebirge ruhig und mit der nötigen Geduld zu beobachten, um zu verstehen, „was der Berg mit uns vorhat". Wie Golser sehr treffend feststellt, wird das heute zunehmend schwierig, wenn man als Berater in einem Tross von "Adabeis" durch den Tunnel getrieben wird und unmittelbar im Anschluss treffsichere Aussagen machen soll. 


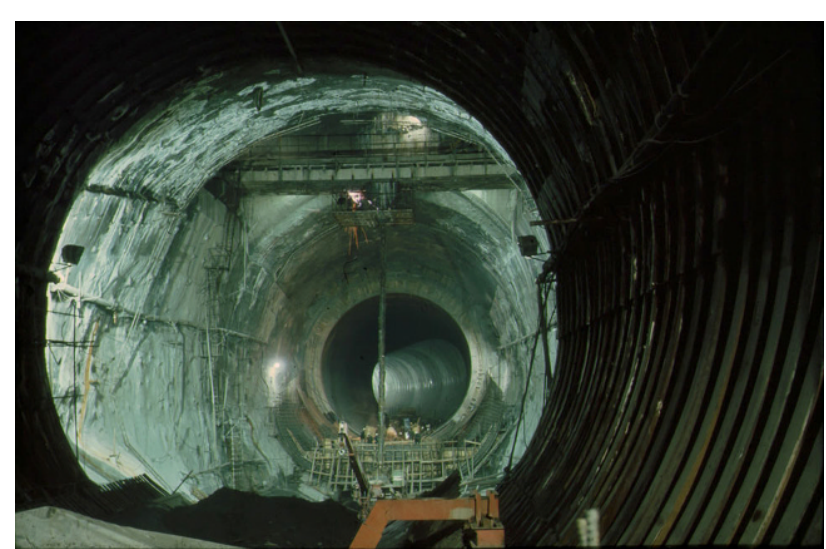

Abb. 5: Tarbela: Schieberkaverne und Schieberschacht

Gleichzeitig mit dem Wolfsbergtunnel war eine Alternativlösung für große Schieberkavernen der Tunnel des Tarbela-Damm-Projektes in Pakistan zu entwickeln. Der US-amerikanische Planer sah einen schweren Stahlausbau nach der ASSM vor. Das europäische Firmenkonsortium fand keine praktikable Lösung für den Bau der bis zu $440 \mathrm{~m}^{2}$ großen Kavernen mit der ASSM. Johann Golser fiel die Aufgabe zu, die von Rabcewicz entwickelten Lösungen im Detail zu bearbeiten.

In Begleitung seiner Frau und der damals drei kleinen Kinder war er dann von 1969 bis 1972 beim Tarbela-DammProjekt in Pakistan, dem damals weltgrößten Bewässerungs- und Kraftwerksprojekt, als Konsulent für Tunnel- und Felsbau tätig (Abb. 4). Dort wurden erstmals unter schwierigen geologischen Bedingungen Hohlräume bis $440 \mathrm{~m}^{2}$ Querschnittsfläche nach den Grundsätzen der NATM aufgefahren und direkte Vergleiche mit dem Stahlausbau nach der ASSM/American Steel Support Method bei Tunnels mit $18 \mathrm{~m}$ Durchmesser möglich. Dort, am Fuß der Vorberge des Himalaya, erlebte Johann Golser nach eigenen Angaben seine schönsten Berufsjahre; es waren wohl auch die bereicherndsten Jahre für die gesamte Familie, denn wann ist ein Bauingenieur schon länger zuhause? Bei diesem Projekt konnten wertvolle Erkenntnisse gewonnen werden, die in zahlreichen Publikationen veröffentlicht und zugleich Anlass zu Golsers Dissertationsthema wurden. Die in Tarbela gemachten Erfahrungen und die Zusammenarbeit mit anderen international tätigen Bau- und Planungsfirmen sollten seinen weiteren Werdegang entscheidend prägen. Die damals größte Baustelle der Welt war für Golser ein Schlüsselerlebnis und zugleich der Anstoß für seine weiteren weltweiten Tätigkeiten. (Abb. 5).

1973 gründete er mit Herrn Dipl.-Ing. Hackl das Ingenieurbüro GEOCONSULT, nachdem sein Chef zum damaligen Zeitpunkt nicht außerhalb des deutschsprachigen Raumes tätig sein wollte. Es bestand auch keine Notwendigkeit, die Auftragslage war damals exzellent. Golser und Hackl aber packte das Fernweh. (Abb. 6).

Von 1974 bis 1983 hielt sich Johann Golser viel im fernen Ausland auf und hatte das Glück, meist zu sehr anspruchsvollen Problemen des Untertagebaues als Experte gerufen zu werden. Länger andauernde Aufenthalte in Japan, Korea, Taiwan, Hongkong und den USA, wo es galt,

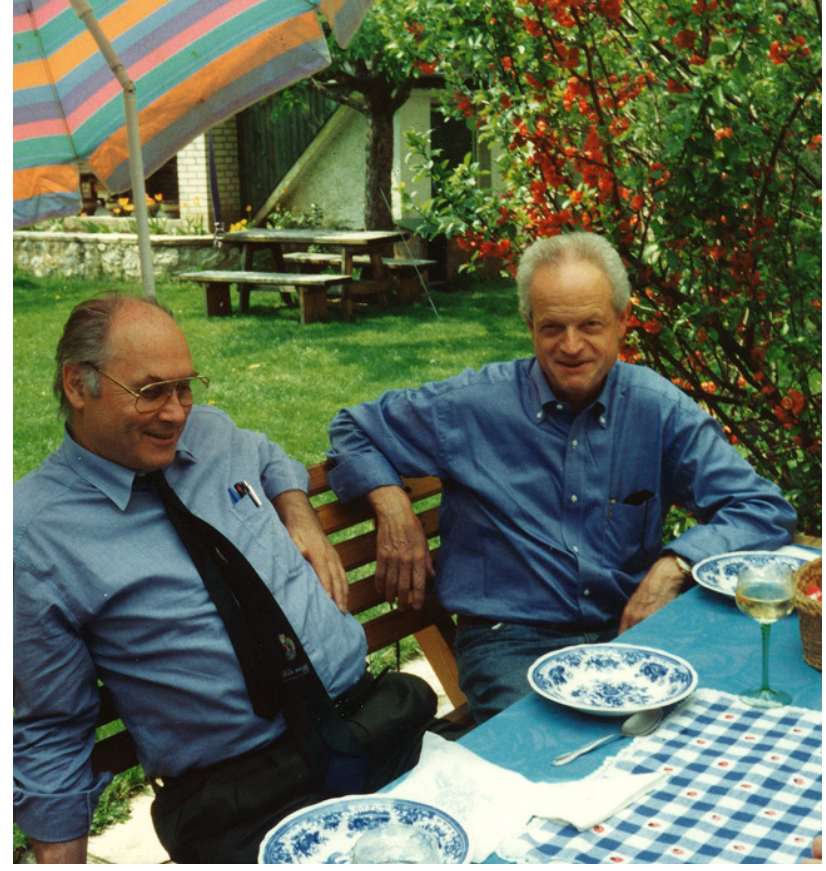

Abb. 6: Johann Golser und Erich Hackl gründen die GEOCONSULT

die NATM für großangelegte Verkehrswegebauten einzuführen, legten den Grundstein für die heute anerkannte Planungs- und Konsulententätigkeit von GEOCONSULT im asiatischen Raum, wie auch auf allen anderen Kontinenten. Zahlreiche Weiterentwicklungen, die die Neue Österreichische Tunnelbaumethode in extrem schwierige geologische und geotechnische Verhältnisse vordringen ließ, stammen aus dieser Zeit. In Deutschland wurde die bis dahin übliche Deutsche Kernbauweise durch die NATM abgelöst. Bauverträge luden Baufirmen dazu ein, Alternativvorschläge anzubieten. Der Salzburger Kreis engagierte sich erfolgreich und so konnte Golser zahlreiche NATM Lösungen ausarbeiten und während des Baus auch begleiten. Im Zuge dieser Arbeiten konnte Golser erstmals eine Ulmenstollen-Lösung vorschlagen, die sich aus der Deutschen Kernbauweise leicht ableiten ließ. Damit konnte das Problem des raschen Ringschlusses und das großer Setzungen infolge des vorher üblichen Kalotten-Strossenvortriebes insbesondere bei sehr breiten Querschnitten besser gelöst werden.

Eine andere Lösung für das rasche Ringschlussproblem wurde erstmals mit der temporären Kalottensohle bei der Giesinger Hangauffahrt in München gefunden. Die Idee kam Golser nach seinem ersten Beratungsauftrag beim Seikantunnel in Japan, wo mit schwerem Stahlrohrausbau ein Kalottenringschluss hergestellt wurde. Der Prüfer für das Los Giesinger Hangauffahrt war Prof. Kupfer von der TU München. Nach Vorstellung der Lösung von Golser nahm Prof. Kupfer seinen Rechenschieber, schrieb eine Formel auf und sagte zu Golser: „Junger Kollege, Ihre Lösung funktioniert nicht." Golser antwortete ihm, dass es das nicht gibt, und wohl in der Berechnung etwas falsch sein müsste. Nach einigen Momenten der Totenstille teilte Prof. Kupfer mit, dass er sich die Lösung nochmal ansehen würde und ein paar Tage später gab er sein Okay. Anlässlich einer 


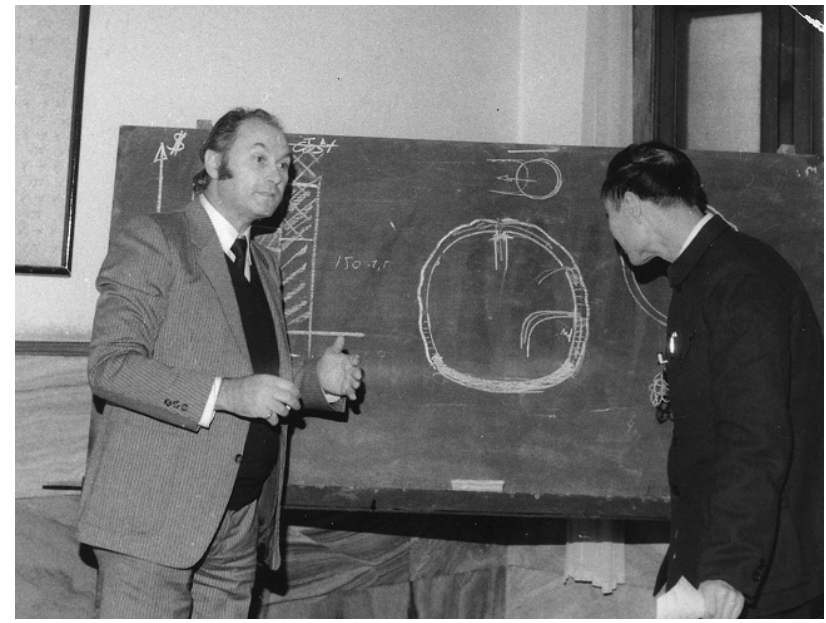

Abb. 7: Johann Golser erläutert Auffahrkonzepte von Tunnelbauwerken

Akademischen Feier in Leoben, wo Prof. Kupfer als Rektor der TU München eingeladen war, erinnerte er sich noch gut an den jungen Ingenieur Golser aus Salzburg. (Abb. 7).

Golser hatte auch den Vorschlag gemacht, systematisch in Kies zu ankern, Selbstbohranker gab es damals allerdings noch nicht. Aus Gesprächen mit seinem Freund Gruber, dem damaligen Chef von GD-Anker in Kärnten, dem Golser seine ersten Kontakte nach Japan verdankte, ergab sich der Anstoß, dass die Firma GD-Anker die ersten Selbstbohranker entwickelte; ein Produkt, das heute weltweit erfolgreich eingesetzt wird.

Nach vielen langen Diskussionen mit japanischen Bauherren für Eisenbahn und Straßenbau war der Boden für den versuchsweisen Einsatz der NATM aufbereitet. So konnten unter praktischer Mithilfe unserer Ingenieure mehrere schwierige Tunnelbauvorhaben, bei denen konventionelle Stahlbauten versagten, erfolgreich bewältigt werden. Die Arbeiten in Japan waren sehr angenehm, waren die Japaner doch sehr lernwillig, kooperativ und vertragstreu.

Weiter ging es in den Fernen Osten im gleichen Stil nach Taiwan, Hongkong und Korea, wo überall U-Bahnen, Straßen- und Eisenbahntunnels gebaut wurden. Die umfangreichen Arbeiten für mehrere Baulose der U-Bahn in Seoul, die vor den Olympischen Spielen im Jahr 1988 fertig sein mussten, wurden von Wulf Schubert als damaliger Mitarbeiter von Geoconsult bravourös geleitet. (Abb. 8).

In Indien wurde die NATM beim Loktak-Hydel-Projekt im äußersten Nordosten im Manipur State erstmals angewandt. Das Projekt wurde nach einer schweren Methangasexplosion zwei Jahre lang eingestellt. Nach Umplanung von ASSM zu NATM wurde der Bau im Jahr 1978 wieder aufgenommen und der Tunnel unter praktischer Anleitung von Mitarbeitern von Golser mit schlagwettergeschütztem Roadheader AM50, einer Maschine, die damals für die Inder neu war, und unter guter Bewetterung fertiggestellt. Der Umstieg von konventionellen Stahlausbauten zur NATM geht in Indien sehr langsam, das Trägheitselement von Entscheidungsträgern ist sehr groß.

In den USA währte der Widerstand gegen die NATM und das Festhalten an der ASSM am längsten. Der erste NATM

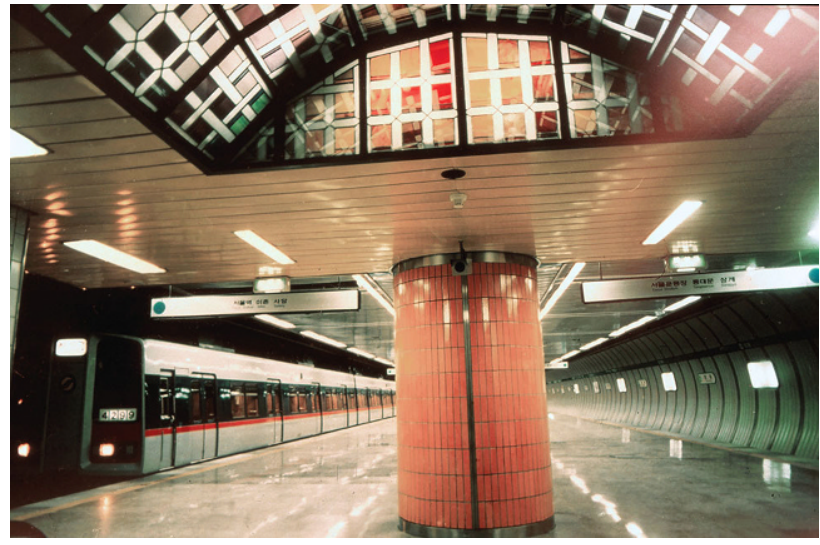

Abb. 8: Seoul: Station Shopping Center Myoung Dong

Tunnel war 1981 der Mt. Lebanon-Tunnel in Pittsburgh. Die NATM-Lösung entstand im Rahmen einer Forschungsfinanzierung in Konkurrenz zu einer lokalen konventionellen Planung. Beide Lösungen wurden ausgeschrieben, der Zuschlag erfolgte zugunsten der wesentlich preisgünstigeren NATM. Das lag wohl auch an der Ausführung durch eine erfahrene österreichische Baufirma. Es folgten U-BahnBaulose in Washington D.C.; mittlerweile ist die NATM als konventionelle Baumethode auch in den USA bestens etabliert.

1984 folgte Johann Golser dem Ruf als Professor an die Montanuniversität Leoben und leitete dort bis zum Jahr 2006, also 22 Jahre lang, das Institut für Geomechanik, Tunnelbau und Konstruktiven Tiefbau als Nachfolger von Prof. Georg Feder. Nicht nur an der Universität hat es Golser geschafft, komplizierte Sachverhalte auf den Punkt zu bringen. Leitgedanke in seinen Vorlesungen war: zuerst Vereinfachen und Abschätzen und erst danach Details bearbeiten. Größenordnungen und grundsätzliches Verständnis für die Aufgaben schaffen die Basis für die Lösung jeglicher Probleme in der Geotechnik und im Tunnelbau. Mit seiner Liebe zur Technik forderte er von seinen Mitarbeitern viel; als Ergebnis seines Wirkens an der Montanuniversität können wir heute auf 13 Dissertationen zurückblicken. Als Rezept für seinen Erfolg gibt Golser an, dass man in jeder Situation versuchen sollte, seine Arbeit richtig und gewissenhaft zu machen. Manchmal gibt es dann im Leben Stationen und Kreuzungen, an denen man die richtige Entscheidung treffen muss. In diesen Momenten spielen Intuition, aber auch ein bisschen Glück eine große Rolle. (Abb. 9).

Schwerpunkte der Forschungstätigkeit von Prof. Golser waren die Weiterentwicklung der NATM, gebirgsmechanische Herausforderungen in einigen Bergbaubetrieben, die Dimensionierung und Auslegung von Salz-Lösungskavernen, der Deponiebau und die Altlastensanierung. Im Zusammenhang mit der Weiterentwicklung der NATM wurden vor allem nachstehende Themen bearbeitet:

- zeitabhängiges Verhalten von jungem Spritzbeton,

- zeitabhängiges Gebirgsverhalten und Langzeitbeanspruchung des Innenausbaus, 


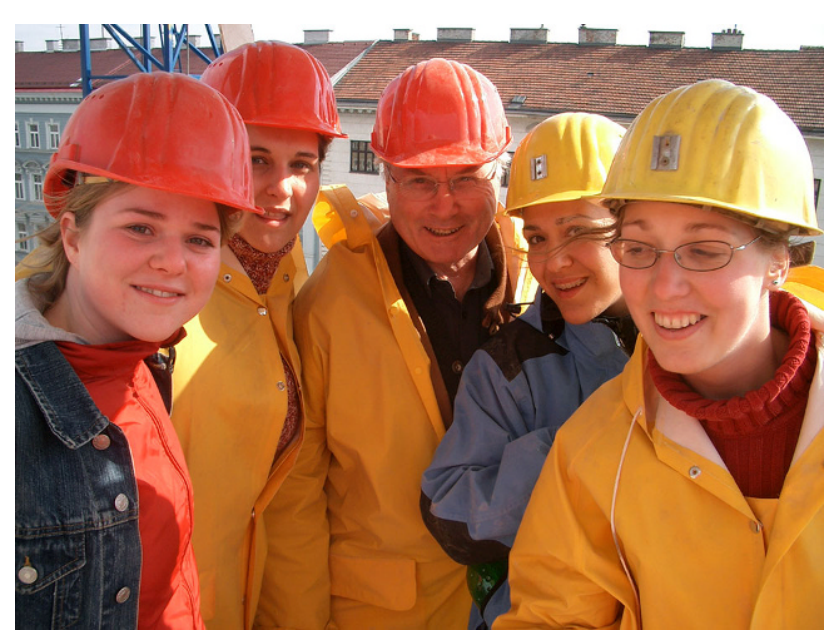

Abb. 9: Exkursionzu Tunnelbaustellen Österreichs, mit Prof. Golser, 2005

- messtechnische Überprüfung der Spritzbetonbeanspruchung mittels Dehnungsmessungen und die Entwicklung eines Messgebers mit Dehnungsmessstreifen, die Initialzündung für das Start-up-Unternehmen GEODATA in Leoben. Es konnte nachgewiesen werden, dass die bis zu diesem Zeitpunkt verwendeten hydraulischen Druckmessdosen für die Ermittlung von Umfangsspannungen in einer Spritzbetonschale ungeeignet sind,

- einschaliger Tunnelausbau,

- erstmalige Entwicklung einfacher und kostengünstiger Stauchelemente in Längsschlitzen der Spritzbetonschale. Beim Tauerntunnel wurden Längsschlitze ohne Stauchelemente vorgesehen, um die Zerstörung der Spritzbetonschale infolge zu großer Stauchungen zu vermeiden, mit dem Nachteil, dass damit auch der Ausbauwiderstand der Schale stark reduziert wurde. Mit der Entwicklung dieser Stauchelemente und dem ersten Einsatz beim Galgenbergtunnel in Leoben wurden diese in der Folge bei vielen Tunnels in stark druckhaften Strecken erfolgreich angewendet und damit das Beanspruchungsvermögen der Spritzbetonschale wieder voll ausgenützt.

Die laufende Konfrontation mit aktuellen Fragestellungen aus der Praxis ermöglichten dem Institut eine sehr anwendungsorientierte Forschung.

Mit der innigen Einbindung in das österreichische Tunnelbaugeschehen war Prof. Golser auch mit bauvertraglichen Konflikten hinsichtlich Vergütung von Bauleistungen befasst. So wurde die von ihm vorgeschlagene Vergütung des Ausbruchs unter Berücksichtigung des Zeitaufwandes für die Sicherungsarbeiten in Form einer Matrix in die ÖNORM B2203 aufgenommen.

Bis zur Gründung des Instituts für Felsmechanik und Tunnelbau an der TU Graz im Jahr 1992 wurden die Vorlesungen zu diesem Thema von Golser als Gastprofessor gehalten. Ferner war Prof. Golser beim Studiengang Bauingenieurwesen der FH Spittal in Kärnten im Entwicklungsteam tätig und widmete sich dort als Vortragender den Fragen des Deponiebaus.

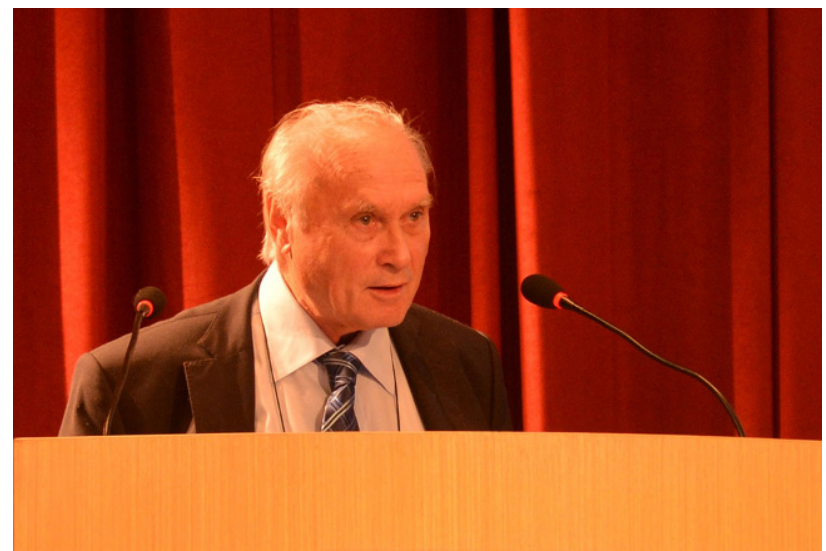

Abb. 10: Seminar zum Thema NATM, Sao Paulo, 2014

In die Zeit seiner Aktivitäten an der Montanuniversität Leoben fiel auch der International Tunnelling Association World Tunnel Congress ITA-WTC 1997, den er als Präsident der Tagung leiten durfte.

Breiten Raum nahmen viele Seminar- und Trainingssessions im fernen Ausland ein, in denen Prof. Golser versuchte, die wichtigsten Grundsätze des Tunnelbaus zu vermitteln. Die erfolgreichsten Veranstaltungen waren jene, in denen Theorie, praktische Anleitung und Baustellenbesuche kombiniert werden konnten. Die Bemühungen wurden belohnt und aus vielen aus dem traditionellen Stahlausbau kommenden Praktikern wurden begeisterte NATM-Anhänger.

Insbesondere in asiatischen Ländern wurden Baustellenbesuche von Bauherrenvertretern gut vorbereitet; im Tunnel sollte es sauber sein, ohne Pfützen, nur der rote Teppich fehlte noch. Es löste großes Erstaunen aus, wenn Prof. Golser, hochgehoben vom Bohrwagen, den Arbeitern vorführte, wie man in der Firste SN-Anker richtig einbaut. Solche praktischen Fertigkeiten hatte sich Prof. Golser in jungen Jahren in Tarbela angeeignet.

Weitere interessante Herausforderungen ergaben sich mit den Aufgaben als Teil von Expertengruppen. Dazu gehörten beispielsweise große Wasserkraftanlagen in Kolumbien, das kürzlich in Betrieb gegangene Neelum-Jhelum Hydel-Projekt in Pakistan, drei verschiedene Trassenführungsstudien für hunderte von Kilometern neuer Eisenbahnstrecken in den Bergen im Norden von Indien sowie ein U-Bahn Projekt in Sao Paulo, das im Zuge politischer Wirren in Brasilien im Moment stillsteht. (Abb. 10) Pakistan wurde seit Tarbela fast zu seiner zweiten Heimat. Ein großes Projekt in über $2000 \mathrm{~m}$ Höhe mit Tunneln, Brücken, Lawinenverbauten und schweren Stützbauwerken ist nach fast einem halben Jahrhundert mit vielen konzeptuellen Änderungen und Unterbrechungen immer noch nicht ganz fertiggestellt und bereitet in der seit Jahren angespannten politischen Situation immer noch Kopfzerbrechen. Nach Empfehlungen von Johann Golser folgten die ersten bergmännischen U-Bahnstationen inmitten der Altstadt von Delhi sowie lange Straßen- und Eisenbahntunnels in Jammu-Kashmir. 


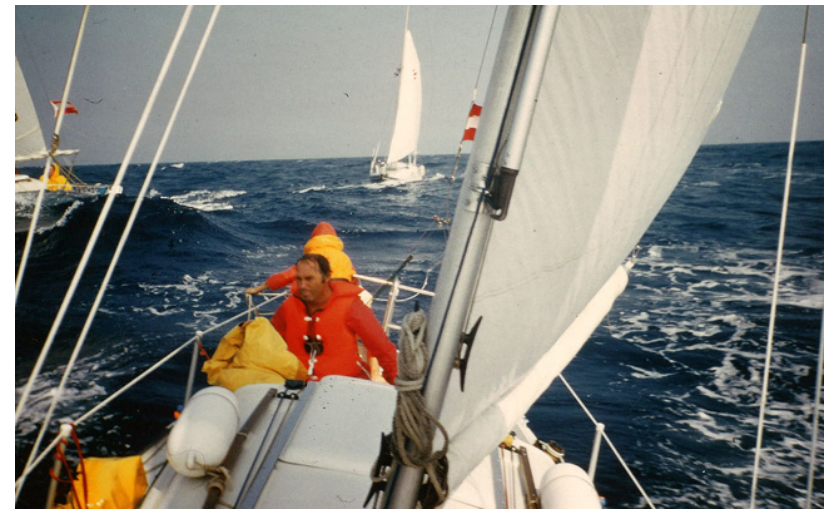

Abb. 11: Johann Golser und sein Hobby, das Segeln

Immer noch bemüht sich Prof. Golser, zum Finden guter Lösungen im Untertagebau etwas beizutragen, es liegt aber jetzt wohl an unserer Generation und den Nachfolgegenerationen, Materialien und Geräte sowie die Möglichkeiten der Digitalisierung, der Datenerfassung und -auswertung bis hin zur Künstlichen Intelligenz weiter zu entwickeln und zu nutzen, damit die NATM weiterhin neu bleiben wird.

Die Liebe zu Land und Leuten in den Bergen des Himalayas und des Hindukush führten Prof. Golser schon vor 50 Jahren durch Afghanistan und später zu Reisen und Trekkingtouren nach Nepal, Kashmir, Ladakh, Sikkim und Tibet. Mit zunehmendem Alter wurden es ausgedehnte Schiffsreisen um den Globus, um an das Hobby des Segelns in den 70-er und 80-er Jahren im Mittelmeer und im Revier Südengland und Kanalinseln anzuschließen. (Abb. 11).

Als Nachfolger von Prof. Golser an der Montanuniversität Leoben habe ich persönlich von ihm die Liebe zum Untertagebau kennengelernt. Er hat mich gelehrt, dass sich der gute Tunnelbauer immer noch dadurch auszeichnet, dass er intuitiv das Richtige macht, indem er mit der Natur und nicht gegen die Natur arbeitet. Mit dem Postulat "mit und nicht gegen die Natur", das von Paracelsus, einem der größten Naturforscher stammt, drängt sich der Vergleich mit der Medizin auf. Golser hat uns gelehrt, dass Menschen wie das Gebirge sind; nicht genormt, inhomogen und anisotrop. Was für den Mediziner das Fieberthermometer ist, sind für den Tunnelbauer die Messungen und Beobachtungen untertage. Auch in der Medizin führt nur der Weg über die Beobachtung zum Befund, zur Diagnose und erst von hier

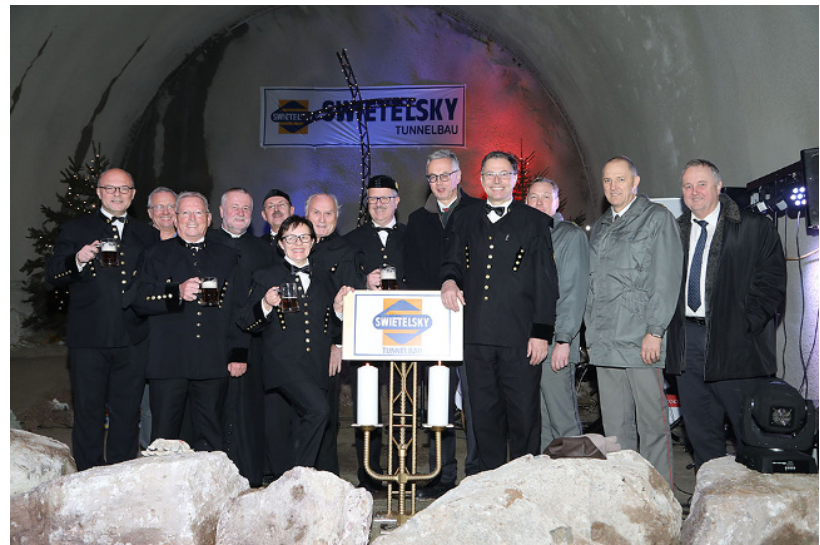

Abb. 12: Prof. Golser zu Besuch der Montanuniversität Leoben im Rahmen der Barbarafeier am 04.12.2017 am ZaB-Zentrum am Berg, einer Untertage-Forschungs- \& Entwicklungs-sowie Trainings-Ausbildungsanlage für den Untertagebau

zur Therapie, welche hoffentlich zum Ziel führt. Es handelt sich somit um eine empirisch-wissenschaftliche Vorgangsweise, womit wir Tunnelbauer uns mit den Medizinern in guter Gesellschaft befinden.

Auch heute noch stellt sich weiterhin die Frage, was wir mit Rechenergebnissen, insbesondere im tiefliegenden Tunnelbau, anfangen können, um die Frage der Sicherheit beantworten zu können. Nach wie vor ist die Methode der Finiten Elemente dazu schlecht geeignet, da die Aufgabenstellung ja schon damit beginnt, die zulässigen Beanspruchungen richtig einzuschätzen und es gilt weiterhin, je mehr man nachdenkt, desto mehr ungelöste Fragestellungen tun sich auf, an welchen wir als Nachfolger von Prof. Feder und Prof. Golser nun beständig weiter arbeiten. (Abb. 12).

Im Namen des Lehrstuhls für Subsurface Engineering, wie das ehemalige Institut für Geomechanik, Tunnelbau und Konstruktiven Tiefbau mit der Emeritierung von Prof. Golser an der Montanuniversität Leoben umbenannt wurde, möchte ich mich bei Prof. Golser als hervorragenden Lehrer und Mentor für seine nationalen und internationalen Aktivitäten im gesamten Fachgebiet des Untertagebaus und seine Forschungsaktivitäten an der Montanuniversität Leoben sehr herzlich bedanken und ihm herzlich zu seinem 80-sten Geburtstag gratulieren!

Wir wünschen dir, lieber Hans, Alles Gute und ein herzliches Glückauf!

Robert Galler 